Research Paper

\title{
Non-Stimulation Needle with External Indwelling Cannula for Brachial Plexus Block and Pain Management in 62 Patients Undergoing Upper-Limb
}

\section{Surgery}

\author{
Bin Yu, Xiaoqing Zhang ${ }^{\bowtie}$, Peili Sun, Shuqi Xie, Qiying Pang \\ Department of Anesthesiology, Tongji Hospital of Tongji University, Shanghai 200065, China.
}

$\triangle$ Corresponding author: Department of Anesthesiology, Tongji Hospital of Tongji University, Shanghai 200065, China; Tel: +86-021-66111509; Fax: +86-021-66111514; E-mail: yubin@tongji.edu.cn; xq_820175@sina.com;

(c) Ivyspring International Publisher. This is an open-access article distributed under the terms of the Creative Commons License (http://creativecommons.org/ licenses/by-nc-nd/3.0/). Reproduction is permitted for personal, noncommercial use, provided that the article is in whole, unmodified, and properly cited.

Received: 2012.08.09; Accepted: 2012.10.15; Published: 2012.10.24

\begin{abstract}
Objective: To investigate the feasibility of a non-stimulation needle with an external indwelling cannula for upper-limb surgery and acute postoperative pain management. Methods: 62 patients undergoing either scheduled or emergency upper-limb surgery received brachial plexus block of modified interscalene or axillary brachial and then postoperative patient-controlled analgesia (PCA) with local analgesics using a specially designed non-stimulation needle with an external indwelling cannula. The outcome measurements included anesthetic effect, acute or chronic complications, postoperative analgesic effect and patient's satisfaction. Results: The success rate of anesthesia was $96.8 \%$. The single attempt placement with the external indwelling cannula was achieved in $85.2 \%$ of patients with axillary brachial plexus block and $78.8 \%$ with modified interscalene brachial plexus block. The incidence of severe intoxication was $3.7 \%$ with axillary brachial plexus block and $3.0 \%$ with modified interscalene brachial plexus block. No hematoma at the injection site, Horner's syndrome, hoarseness or dyspnea was observed. Postoperative analgesic effect was achieved in $100 \%$ and activities were slightly lowered in $91.7 \%$. The incidence of nausea and vomit was 8.3\%; patient's satisfaction was 9.1 on a 10 -point scale system. Infection, nerve injury and respiratory depression were absent during the catheter indwelling. The indwelling time of external indwelling cannula was $30.5 \mathrm{~h}$ on average. There was no nerve injury related complication after withdrawing the external indwelling catheter. Conclusions: Brachial plexus block using a non-stimulation needle with an external indwelling cannula has favorable intra-operative anesthetic benefit and provides an excellent postoperative analgesic outcome. The low incidence of complications and favorable patient's satisfaction suggest that non-stimulation needle with an external indwelling cannula is a useful and safe anesthetic tool in brachial nerve block and acute postoperative pain management.
\end{abstract}

Key words: non-stimulation needle, indwelling cannula, brachial plexus block, postoperative pain management

\section{Introduction}

In current clinical practice, the insertion of a perineural catheter is performed either through the internal lumen of a nerve stimulation needle or through a plastic cannula using the cannu- la-over-needle technique. However, it is invalid to accurately orient the flexible catheter tip [1] and usually results in serious complications [2]. Using a stimulatory catheter tipped with a nerve stimulator makes 
the procedure more complicated. Moreover, continuous postoperative infusion of analgesics may cause leakage, due to the residual space between the tunnel formed after withdrawing of stimulation needle or cannula and the internal lumen of the flexible catheter. The catheter used in the cannula-over-needle technique with a plastic cannula often has a subcutaneous tunnel of $4-5 \mathrm{~cm}$, which further increases the risk for infection $[3,8]$. In this study, a specially designed non-stimulation needle with an external indwelling cannula was used for brachial plexus block. It contains a stainless-steel, solid cannula needle and a short needle tip with a $30^{\circ}$ bevel. The external cannula is composed of biocompatible polyurethane, and the side holes in the tip allow the smooth infusion. The external cannula is labeled with a radiologically opaque scale, allowing the placement of the cannula and the infusion under the radiography. It is useful for locating the cannula in case of accidental fracture. When the needle is corrected in position, the paresthesias or facial pop methods may be present, the needle should not be moved, and its external cannula may be inserted forward $2-3 \mathrm{~cm}$, then the needle was pulled out and the external cannula fixed to the skin with a medical tape. With this technique, patients can receive local anesthesia intra-operatively and patient-controlled analgesia postoperatively via the cannula. Thus, the cannula can be fixed immediately after the peripheral nerve block. This protocol does not require changing the catheter. This study aimed to investigate the efficacy of anesthesia and postoperative analgesia and to evaluate the adverse effects and patient's satisfaction using the specially designed non-stimulation needle with external indwelling cannula. Our findings may provide evidence on the feasibility of this technique in clinical brachial plexus block and postoperative pain management.

\section{Patients and Methods}

\section{Patient enrollment}

The study was approved by the Institutional Review Board at Tongji Hospital of Tongji University. Informed consent was obtained prior to enrollment from each patient. Sixty-two adult patients aged 18-65 years (median: 44 years, male/female: 38/24) who received scheduled or emergency surgery were recruited and underwent brachial plexus block of modified interscalene or axillary brachial plexus block using a specially designed non-stimulation needle with an external indwelling cannula in an anatomical location or the tunical penetration protocol, followed by postoperative patient-controlled analgesia (PCA) with $0.2 \%$ ropivacaine in this study. The brachial plexus block of modified interscalene was used in cases with fracture involving the clavicle, proximal humerus, shoulder, forearm or palm dominated by radial nerve, whereas axillary brachial plexus block was performed in cases receiving surgery on the elbow, forearm or palm dominated by median and ulnar nerve. Exclusion criteria included allergy to amide local anesthetics, serious cardiac, pulmonary, liver or renal dysfunction, a known history of opioid allergy and/or abuse and general anesthesia as a result of an unacceptable nerve block outcome.

\section{Anesthetic procedures}

The blocks were performed by 2 anesthetic attending physicians with above 10-year clinical experiences. Intramuscular atropine $(0.5 \mathrm{mg})$ and phenobarbital $(0.1 \mathrm{mg})$ were given $30 \mathrm{~min}$ prior to anesthesia. In the operation room, the patients received routine noninvasive monitoring of blood pressure, pulse, oxygen saturation and electrocardiograph (ECG). Moreover, oxygen was delivered at $2 \mathrm{l} / \mathrm{min}$ while access to the median cubital vein was established. The brachial plexus block using a specially designed non-stimulation needle with an external indwelling cannula (22-gauge needle, 50-mm-length external indwelling cannula; Tuoren Group, Xinxiang, China) (Figure 1). The brachial plexus block in the present study was performed via the Borgeat's interscalene brachial plexus block [3] (Figure 2) or axillary brachial plexus block (Figure 3) with modification depending upon the type of surgery. Using the modified Borgeat's interscalene brachial plexus block, the puncture needle was positioned at a small angle relative to the long body axis and in an appropriate orientation, thus minimizing the bending and occlusion of the catheter when the patient's neck moved. When the non-stimulation needle was in a corrected position, paresthesias or facial pop metheds were present, the needle did not move, and its external cannula moved forward 2-3 cm, then the needle was pulled out and the external cannula fixed to the skin with no-pressure medical tape. Following the injection at the axillary sheath, a spindle-shaped bulge was present along the axis of the axillary sheath. The local anesthetics were used intra-operatively including $2 \%$ lidocaine $(20 \mathrm{ml})$ plus $0.75 \%$ ropivacaine $\mathrm{HCl}(10 \mathrm{ml})$. The patients achieving favorable anesthesia received preoperative sedation by intravenous bolus infusion of droperidol $(2.5 \mathrm{mg})$ and fentanyl $(0.05 \mathrm{mg})$.

Upon the completion of surgery, all patients received PCA through the indwelling external cannula. The analgesic consisted of $60 \mathrm{ml}$ of $0.75 \%$ ropivacaine in $200 \mathrm{ml}$ of normal saline: a loading dose was $5 \mathrm{ml}$, 
followed by continuous infusion at a rate of $4 \mathrm{ml} / \mathrm{h}$ and a bolus injection of $3 \mathrm{ml}$ within $20 \mathrm{~min}$. In the event of an unacceptable postoperative analgesic outcome [a visual analogue scale (VAS) score > 6], a single 100-mg of bucinnazine was administered intramuscularly.



Figure I Non-stimulation needle with external indwelling cannula used in this study.
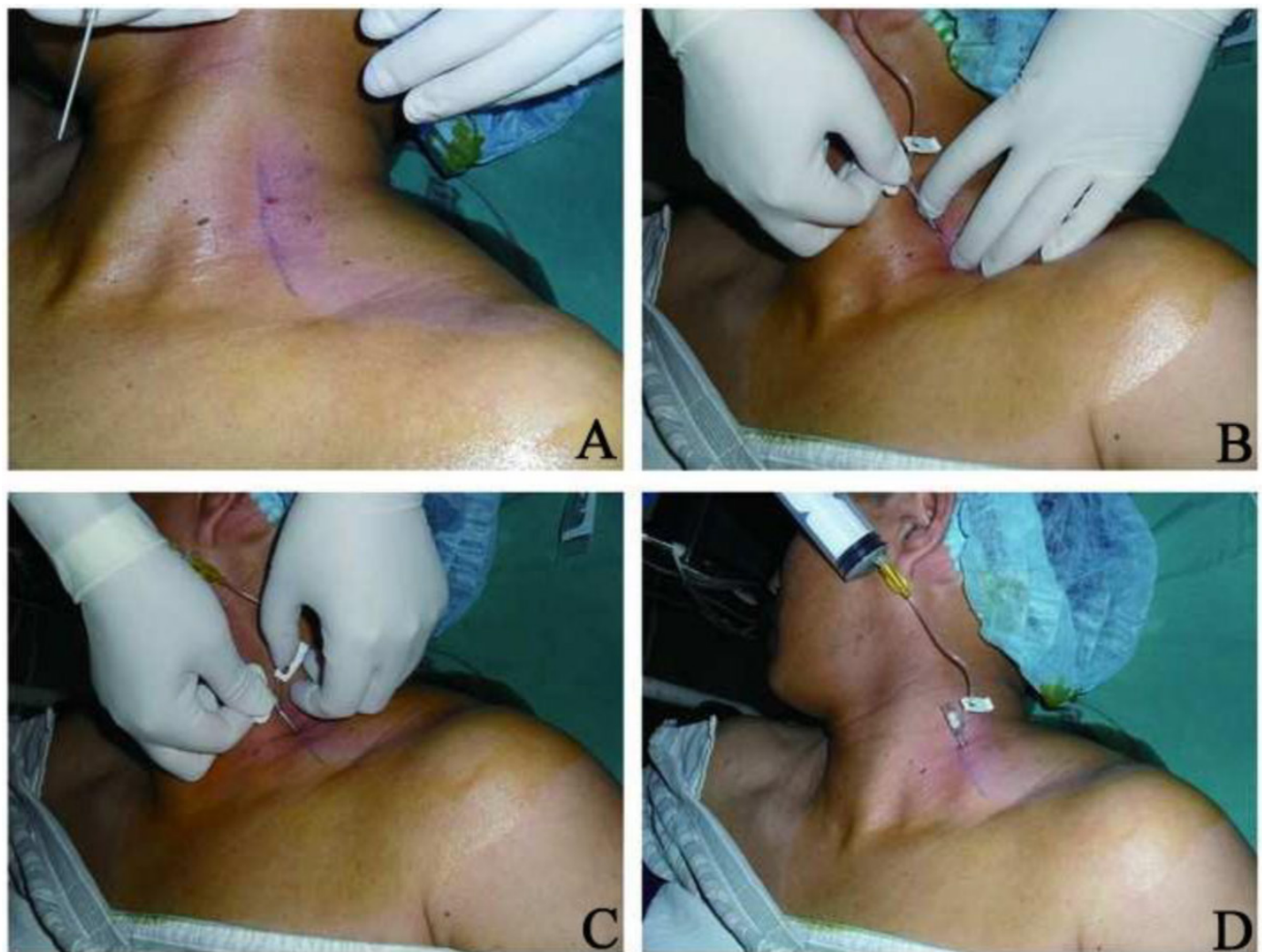

Figure 2 Brachial plexus block by modified interscalene cannulation A: Positioning along the interscalene line; B: Insertion of catheter for nerve block; C: Insertion of external external; D: Withdrawing of core needle and indwelling of external cannula. 

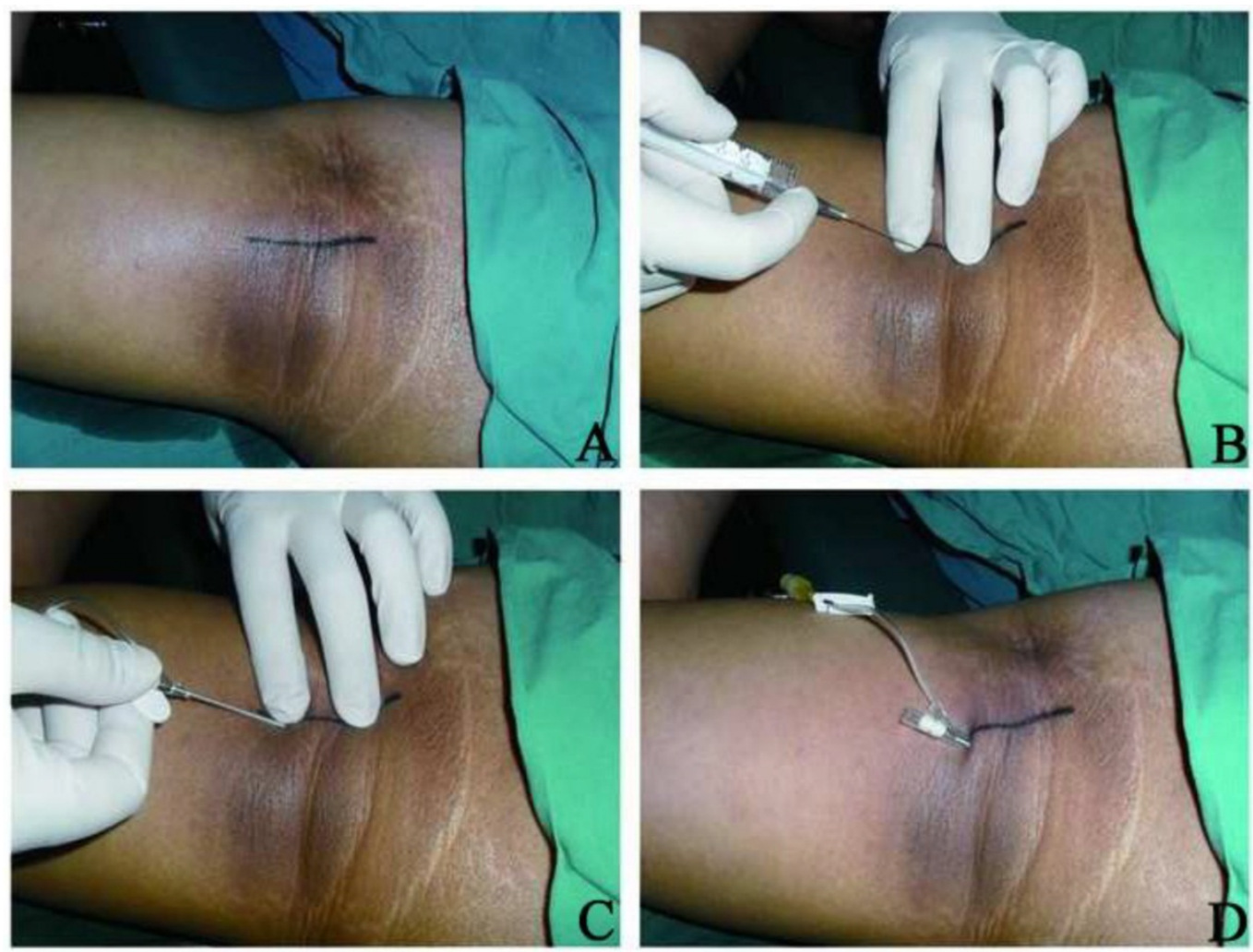

Figure 3 Brachial plexus block of axillary sheath cannulation. A: Positioning along the middle axillary line; B: Local anesthesia at the puncture site; C: Insertion of catheter for nerve block; D: Withdrawing of core needle and indwelling of external cannula.

\section{Outcome measurements}

Follow-up visits were performed by 2 anesthetic attending physicians with similar qualifications at 2 , $6,12,24$ and $40-48 \mathrm{~h}$ after surgery, as well as 7 days post-surgery. The outcome measurements of this study included: the difficulty in puncture/placement of the non-stimulation needle with external indwelling cannula (over 3 times); the frequency of puncture attempts; anesthetic effect; postoperative analgesic effect; patient's satisfaction; incidence of adverse events such as nausea/vomiting and over-sedation; and puncture site or indwelling cannula-associated infection and nerve injury. Severe intoxication was considered as severe complication. The anesthetic effect was rated as follows: excellent: complete block, pain-free and good muscle relaxation; good: less-favorable block and requiring additional local infiltration block with local intravenous anesthetics; poor: noncooperation with surgery and requiring general anesthesia due to serious pain. The analgesic outcomes were assessed with a 10-cm VAS at 2, 6, 12, 24 and 40-48 h after surgery as follows: $0-2$, no or mild pain; 3-6, moderate but tolerable pain; 7-10, severe and intolerable pain [4]. For each patient, the mean pain score and the frequency of bolus injection as well as the dose of bucinnazine were recorded. The Ramsay Sedation Scale (RSS) was used to assess the sedation as follows: 1), anxious and agitated and/or restless; 2), cooperative, oriented and tranquil; 3 ), responsive to commands only; 4), falling asleep and responding briskly to a light glabellar tap or loud auditory stimulation; 5), falling asleep and responding sluggishly to a light glabellar tap or loud auditory stimulation; and 6, falling asleep and nonresponsive to a light glabellar tap or loud auditory stimulation [5]. A RSS of 2-4 reflects favorable sedation, whereas a RSS of 5-6 indicates over-sedation. Respiratory depression was defined as a respiration rate below 8 breaths per minute. Patients' satisfaction was determined using a 10-point scale, with 0 being the least satisfied and 10 being the most satisfied.

\section{Statistical analysis}

For most measurements in this study, descriptive statistics were applied. The quantitative data were expressed as mean \pm standard deviation (SD) unless otherwise specified.

\section{Results}

A total of 62 patients were enrolled into this study. Among them, 2 patients requiring general an- 
esthesia were excluded because of the changes in medical conditions. The brachial plexus block was considered successful when preoperative testing showed no pain at the surgical site. 60 patients successfully underwent upper limb surgery according to the treatment plan. The anesthetic methods are shown in Table 1 and the situations and complications in Table 2. Severe intoxication was found in $3.7 \%$ of patients receiving axillary brachial plexus block and $3.0 \%$ of patients receiving modified interscalene brachial plexus block. The patient complained of the ceiling moving as a wave at $15 \mathrm{~min}$ after local anesthesia, which was considered as a severe intoxication symptom. The postoperative data and complications are summarized in Table 3. Five female patients showed post-operative nausea and vomiting. RSS was 0.0 on average. Three patients complained of paresthesia at the affected upper limb or thumb. Then, the PCA infusion was slowed to a rate of $2 \mathrm{ml} / \mathrm{h}$ and the paresthesia resolved. One patient complaint of post-injection head and neck stiffness, which resolved after intramuscular injection of $10 \mathrm{mg}$ of valium. Another patient complained of hearing loss and transient diplopia which disappeared after the PCA infusion was slowed to a rate of $1-2 \mathrm{ml} / \mathrm{h}$. No patient developed a hematoma at the injection site, Horner's syndrome, hoarseness or dyspnea. No patient experienced the respiratory depression, and drug leakage was not observed during the post-surgery follow-up period. There were no signs of local infection upon removing the indwelling cannula. No patients complained of paresthesia at the affected upper limb on postoperative day 7 after removal of the cannula. None of the patients received the intramuscular injection of bucinnazine.

Table I. Different anesthetic methods used in patients

\begin{tabular}{ll}
\hline No & 62 \\
Planned axillary cannula & 27 \\
Planned modified interscalene cannula & 30 \\
$\begin{array}{l}\text { Planned modified interscalene cannula }+ \\
\text { general anesthesia }\end{array}$ & 3 \\
Unplanned general anesthesia & 2 \\
\hline
\end{tabular}

Table 2. Situations and complications during placement of external indwelling cannula

\begin{tabular}{lll}
\hline & Axillary cannula & $\begin{array}{l}\text { Modified inter- } \\
\text { scalene cannula }\end{array}$ \\
\hline No & 27 & 33 \\
Single attempt success & $23 / 27(85.2 \%)$ & $26 / 33(78.8 \%)$ \\
Excellent/good anesthesia & $26 / 27(96.3 \%)$ & $32 / 33(97 \%)$ \\
Severe intoxication & $1 / 27(3.7 \%)$ & $1 / 33(3.0 \%)$ \\
\hline
\end{tabular}

Table 3. Postoperative parameters and complications

\begin{tabular}{ll}
\hline $\begin{array}{l}\text { Postoperative sufficient analgesic } \\
\text { (no activities/ activities) }\end{array}$ & $60 / 55$ \\
Postoperative nausea/vomiting & $5 / 60$ \\
RSS & 0.0 \\
Cannula occlusion & $2 / 60$ \\
Cannula slipping & $4 / 60$ \\
Infection at puncture site & 0 \\
Duration of PCA use (h) & 30.5 \\
Nerve injury related complication & 0 \\
Severe intoxication & $3 / 60$ \\
Urinary retention & 0 \\
Patient satisfaction $(0 \sim 10)$ & 9.1 \\
\hline
\end{tabular}

PCA: Patient Controlled Analgesia

\section{Discussion}

Currently, a multimodal analgesia technique is highly recommended to minimize the adverse effects of opioid agents during nerve block [6]. Therefore, continuous brachial nerve block and postoperative analgesia with local anesthetics are being used more frequently and widely. However, the commonly used intra-nerve-stimulation needle or intra-cannula indwelling catheter disrupts the orientation of catheter tip [2] and may cause leakage. When a catheter tipped with a nerve stimulator is used for localization, the anesthetic procedure becomes technically challenging. In this study, the non-stimulation needle with external indwelling cannula can be fixed immediately after the peripheral nerve block because it is unnecessary to change the catheter. Therefore, this needle (characterized by external cannula closely enveloping nerve block needle) allows the simultaneous placement in the nerve block puncture. After being properly oriented, the external cannula is placed directly into the nerve sheath and fixed with no-pressure plastic film, as opposed to the subcutaneous tunnel indwelling. In addition, the continuous peripheral nerve block cannula is less likely to cause leaking of drugs, and the postoperative analgesia requires only a short duration of indwelling, and thus the risk for infection is reduced $[2,7]$. This device is similar to IV cannula in the shape, but truly different regardless of the external indwelling cannula or the pin core. When used for continuous brachial plexus block, IV cannula presents with sharp pin core angle and the sensation of breaking through the nerve sheath is not obvious; thus, nerves are easily injured; moreover, the cannula left is easily obstructed in the anterior end by soft tissues and infusion of local anaesthetics can not be continued post surgery. For the novel device, the pin core and the long axis have an obtuse angle, around 30 
degrees, and the sensation of breaking through the intermuscular sheath and axillary sheath is very obvious; thus, nerves are not easily damaged; furthermore, there are many lateral apertures in the anterior ends of cannula, which ensures the continuous infusion of local anaesthetics post surgery.

Our study demonstrated that the two types of brachial plexus block using the non-stimulation needle with an external indwelling cannula were comparable in the success rate at one attempt (axillary: $85.2 \%$ and modified interscalene: $78.8 \%$ ), anesthetic effect (axillary: $96.3 \%$ and modified interscalene: 97\%) and postoperative analgesia effectiveness (no activities $100 \%$ and activities $91.7 \%$ ) and had favorable safety: low incidence of intraoperative severe intoxication (axillary: 3.7\% and modified interscalene: $3.0 \%)$, nausea/vomiting (8.3\%) and over-sedation. Additionally, the patients' satisfaction was high (9.1). In 4 patients, the indwelling cannula slipped accidentally due to frequent activity. Ekatodramis and Borgeat reported that subcataneous tunnelling of interscalene catheters could prevent the cather dislocation, but increased the risk for infection[9]. The time of external indwelling cannula was $30.5 \mathrm{~h}$ on average in our study. Thus, in our opinion, the continuous peripheral nerve analgesia (CPNA) with adequate indwelling but without tunnelling meets the requirements of postoperative acute analgesia. In 2 patients, the indwelling cannula was occluded due to the compression by the neck. No infection (erythema, swelling or pain at the puncture site), leaking of drugs and nerve injury associated with cannula indwelling were observed in the present study. Although 3 patients complained of paresthesia at the affected upper limb or thumb and 1 patient suffered from hearing loss and transient diplopia, to slow the CPNA infusion resolved these symptoms. The head and neck stiffness in 1 patient also resolved after intramuscular injection of valium $(10 \mathrm{mg})$. We postulated that these symptoms served as toxicity of local anesthesia, rather than nerve injury associated with the non-stimulation needle. Thus, we concluded that the specially designed non-stimulation needle with an external indwelling cannula is feasible for brachial plexus block and postoperative acute pain management. On the basis of our experience, the use of this needle up to 48 $\mathrm{h}$ seems to be safe. Of cause, there is no doubt that application of this needle should be cautious, especially under the ultrasound guidance in future [10].

There were still limitations in this study. No controls were recruited in our study and the sample size was relatively small. However, our findings may provide evidence for future large-scale trials.

\section{Acknowledgements}

We thank Professor Jianguo $\mathrm{Xu}$, Yannan Hang, Sukun Fu and Dr. Frank A. Beger for their kind help in this study. This work was supported by the Combining Learning with Research and Production Fund of 2010 Tuoren Anesthesia Supplies Limited Company.

\section{Competing Interests}

The authors have declared that no competing interest exists.

\section{References}

1. Klein SM, Grant SA, Greengrass RA, Nielsen KC, Speer KP, White W, Warner DS, Steele SM. Interscalene Brachial Plexus Block with a Continuous Catheter Insertion System and a Disposable Infusion Pump. Anesth Analg. 2000; 91(6): 1473-8.

2. Capdevila X, Pirat P, Bringuier S, Gaertner E, Singelyn F, Bernard N, Choquet O, Bouaziz H, Bonnet F. French Study Group on Continuous Peripheral Nerve Blocks. Continuous peripheral nerve blocks in hospital wards after orthopedic surgery: a multicenter prospective analysis of the quality of postoperative analgesia and complications in 1,416 patients. Anesthesiology 2005; 103:1035-45

3. Borgeat A, Dullenkopf A, Ekatodramis G, Nagy L. Evaluation of the lateral modified approach for continuous interscalene block after shoulder surgery. Anesthesiology. 2003; 99(2):436-42.

4. Maxwell C. Sensitivity and accuracy of the visual analogue scale: a psycho-physical classroom experiment. $\mathrm{Br} \mathrm{J}$ Clin Pharmacol. 1978;6(1):15-24

5. Ramsay MAE, Savege TM, Simpson BRJ \& Goodwin R. Controlled sedation with alpaxalone-alphadolone. Br Med J 1974; 2: 656 - 659.

6. Klein SM. Continuous Peripheral Never Blocks: Fewer Excuses. Anesthesiology, 2005; 103: 921-923.

7. Neuburger M, Buttner J, Blumenthal S, Breitbarth J, Borgeat A. Inflammation and infection complications of 2285 perineural catheters: a prospective study. Acta Anaesthesiol Scand 2007; 51:108-14

8. Hebl JR. The importance and implications of aseptic techniques during regional anesthesia. Reg Anesth Pain Med 2006; 31:311-23

9. Ekatodramis G, Borgeat A. Subcutaneous tunneling of the interscalene catheter: A simple and effective method to prevent interscalene catheter dislocation. Can J Anaesth 2000; 47:716-7

10. Tsui B. Ultrasound-guidance and nerve stimulation implications for the future practice of regional anesthesia. Can J Anaesth 2007; 54: 165-170 